\title{
Ion Trap with Narrow Aperture Detection Electrodes for Fourier Transform Ion Cyclotron Resonance Mass Spectrometry
}

\author{
Konstantin O. Nagornov, ${ }^{1}$ Anton N. Kozhinov, ${ }^{1}$ Oleg Y. Tsybin, ${ }^{2}$ Yury O. Tsybin ${ }^{1,3}$ \\ ${ }^{1}$ Biomolecular Mass Spectrometry Laboratory, Ecole Polytechnique Fédérale de Lausanne, 1015, Lausanne, Switzerland \\ ${ }^{2}$ Ion Physics Laboratory, State Polytechnical University, 195251, Saint Petersburg, Russia \\ ${ }^{3}$ Spectroswiss Sàrl, 1015, Lausanne, Switzerland
}

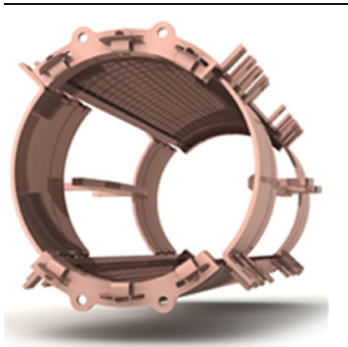

NADEL ICR cell

Abstract. The current paradigm in ion trap (cell) design for Fourier transform ion cyclotron resonance mass spectrometry (FT-ICR MS) is the ion detection with wide aperture detection electrodes. Specifically, excitation and detection electrodes are typically $90^{\circ}$ wide and positioned radially at a similar distance from the ICR cell axis. Here, we demonstrate that ion detection with narrow aperture detection electrodes (NADEL) positioned radially inward of the cell's axis is feasible and advantageous for FT-ICR MS. We describe design details and performance characteristics of a $10 \mathrm{~T}$ FT-ICR MS equipped with a NADEL ICR cell having a pair of narrow aperture (flat) detection electrodes and a pair of standard $90^{\circ}$ excitation electrodes. Despite a smaller surface area of the detection electrodes, the sensitivity of the NADEL ICR cell is not reduced attributable to improved excite field distribution, reduced capacitance of the detection electrodes, and their closer positioning to the orbits of excited ions. The performance characteristics of the NADEL ICR cell are comparable with the state-of-the-art FT-ICR MS implementations for small molecule, peptide, protein, and petroleomics analyses. In addition, the NADEL ICR cell's design improves the flexibility of ICR cells and facilitates implementation of advanced capabilities (e.g., quadrupolar ion detection for improved mainstream applications). It also creates an intriguing opportunity for addressing the major bottleneck in FTMS-increasing its throughput via simultaneous acquisition of multiple transients or via generation of periodic non-sinusoidal transient signals.

Keywords: Fourier transform, FT, Fourier transform mass spectrometry, FTMS, Ion cyclotron resonance, ICR, Transient signal, lon trap, Proteomics

Received: 21 August 2014/Revised: 27 January 2015/Accepted: 29 January 2015/Published Online: 14 March 2015

\section{Introduction}

$\mathrm{F}$ ourier transform ion cyclotron resonance mass spectrometry (FT-ICR MS) provides the highest resolving power and mass accuracy for molecular structural analysis [1-6]. Topdown proteomics and petroleomics, because of their extreme requirements for analytical performance, are the primary application areas of FT-ICR MS nowadays [3, 7]. Over the past decade, other application areas of interest were primarily addressed using Orbitrap FTMS and high-resolution time-of-

Electronic supplementary material The online version of this article (doi:10.1007/s13361-015-1089-y) contains supplementary material, which is available to authorized users.

Correspondence to: Yury Tsybin; e-mail: yury.tsybin@epfl.ch flight (TOF) MS [4, 8-11]. Nevertheless, the extreme analytical challenges in molecular structural analysis require further increase of FTMS performance. Owing to its fundamental power, further development of FT-ICR MS is feasible and may allow bridging the gap between the analytical requirements and MS capabilities. For that, a corresponding increase in high-performance analysis throughput is required. For example, even at the current data acquisition rate, the exceptional FT-ICR MS capabilities trigger development of novel approaches of molecular structural analysis [12]. Among those, the uses of isotopic fine structure information in qualitative bottom-up proteomics and mass defect information in quantitative bottom-up proteomics appear particularly attractive [1315]. Increased, for example, by a factor of $2-10$, data acquisition rate should allow interrogation of more peptides at the required structural level and provide improved qualitative and 
quantitative protein analyses. Development of novel concepts for ion conditioning and manipulation in ICR cells based on improved understanding of ion motion fundamentals has thus become a target of recent innovations in the field $[6,16]$.

Since the inception of FT-ICR MS by Marshall and Comisarow, the design of ICR cells has been primarily based on the use of wide aperture detection electrodes and sinusoidal ion signals (that is, generation of transient signals whose spectral energy is concentrated primarily at the fundamental ion frequency) $[12,17]$. Since the last decade or so, the most common ICR cell configuration has been a cylindrical cell with a pair of $90^{\circ}$-wide excitation electrodes and a pair of similar $90^{\circ}$-wide detection electrodes $[4,18]$. The detected image charge ion signal is averaged during ion motion along the detection electrode surface, requiring low frequency (as determined from Nyquist criteria) sampling. The drawbacks of this configuration include a certain limitation on the acquisition rate, substantial perturbation of excitation radio frequency RF field by detection electrodes, and a low degree of design flexibility.

Marshall and co-workers have recently demonstrated by simulations that $120^{\circ}$-wide excitation electrodes are optimal for coherent ion dipolar excitation and elimination of the third harmonic [19]. However, implementation of such electrodes for ion excitation would reduce the efficiency of ion detection using the currently employed ICR cell design concept. A feasible, although technically challenging, solution to this problem is the use of external switching electronic circuitry allowing for ion excitation and detection using the same pair of $120^{\circ}$-wide electrodes.

Quadrupolar modes of ion excitation, including the quadrature excitation, are other attractive approaches for improving the coherence of excited ion motion [20-23]. ICR cell operation in these modes also suffers from the same low flexibility of the current ICR cell design, when wide aperture detection electrodes compete with the excitation electrodes for space. Improved coherence of ion motion in ICR cells has been efficiently addressed via harmonization of the trapping electric fields by a number of approaches, including those of Tolmachev et al. [24, 25], Gross et al. [26], Bruce et al. [27, 28], and Nikolaev and co-workers [29, 30]. The obtained improvements in FT-ICR MS performance are quite dramatic. Specifically, the transient lifetime has been substantially, about $10-$ to 100 -fold, increased. The dynamically harmonized ICR cell, following the original idea of Boldin and Nikolaev [29], is particularly impressive, with demonstrated resolution of up to 40 million on a peptide (single peak) and a routinely achieved isotopic fine structure resolution on peptide ions even in moderate, $7 \mathrm{~T}$, magnetic fields [31]. The main drawback of the harmonized cells is that they do not improve the throughput (or the acquisition rate) of FT-ICR MS.

Signal detection at multiples of ion (reduced) cyclotron frequency, which is a feasible approach for increasing the throughput [32-36], is also limited by the design of those cells: they also comprise relatively wide aperture excitation and detection electrodes, resulting in a restricted factor of the generated multiples (e.g., $\sim 22^{\circ}$ electrodes and the fourth multiple for a 16-electrode ICR cell). A number of attempts have been made to reduce the aperture of detection electrodes. For example, Nikolaev and co-workers suggested an ICR cell with four detection electrodes made of conducting wires [32]. However, the experimental implementation of the suggested ICR cell was deferred. One of the possible reasons for the failure was the positioning of these electrodes directly below the excitation electrodes in the areas with strong excite RF field, leading to excitation field distortion and pre-amplifier saturation prior to ion detection.

Here, we describe a concept, design, and implementation of an ICR cell with narrow aperture (flat) detection electrodes (NADEL) addressing the limitations of FTICR MS design and capabilities specified above. The analytical characteristics of thus introduced NADEL ICR cells are presented and their particular advantages are outlined.

\section{Experimental}

\section{Sample Preparation}

Substance P, MRFA, Ultramark, and ubiquitin were purchased from Sigma-Aldrich (Buchs, Switzerland). ESI calibration mixture pre-formulated at low concentration was purchased from Agilent (Basel, Switzerland). Serum albumin (bovine) was purchased from Fisher Scientific (Schwerte, Germany). Polyphosphoric acid was obtained from Fluka (Buchs, Switzerland). C-terminally amidated peptides P1, P2, and P3: P1 EESTR (monoisotopic mass 619.29255 Da), P2 - PMMSR (619.29342 Da), and P3 - HDGHR (619.29389 Da) were synthesized in-house with solid state peptide synthesis. All peptide and protein samples were used without further purification. LC-MS grade acetonitrile and water were obtained from Fluka (Buchs, Switzerland). Formic acid was obtained from Merck (Zug, Switzerland). Peptide and protein solutions were prepared in $1: 1(\mathrm{v} / \mathrm{v})$ water/acetonitrile solvent mixtures containing $0.1 \%(\mathrm{v} / \mathrm{v})$ of formic acid. The final sample concentration for all samples was $\sim 1 \mu \mathrm{M}$, except $\sim 10 \mu \mathrm{M}$ for albumin. The polyphosphoric calibration mixture was prepared by adding $1 \%(\mathrm{v} / \mathrm{v})$ of polyphosphoric acid to $1 \mathrm{~mL}$ of water. Maltene fraction of Venezuelan crude oil was dissolved in dichloromethane, followed by 1:23 dilution in acetonitrile with the addition of $2 \%$ of formic acid (by volume), as described previously [9].

\section{NADEL ICR Cell Design and Mass Spectrometry}

The design of the NADEL ICR cell employed here was developed based on the open-ended cylindrical ICR cell (Ultra Cell; Thermo Scientific, Bremen, Germany). The commercially available Ultra cell contains two pairs, inner and outer, of segmented trapping ring electrodes and excitation grids, placed over the entire length of the cell, in addition to the conventional $90^{\circ}$ electrodes (a pair of which are used for ion detection and another pair is grounded) [4]. Ultra cell's inner diameter is of $\sim 56 \mathrm{~mm}$ and length of middle section (detection) electrodes is 
of $\sim 70 \mathrm{~mm}$. In NADEL ICR cell, the standard $90^{\circ}$ detection electrodes were substituted with narrow aperture (flat) detection electrodes with a thickness of $1 \mathrm{~mm}$. Importantly, the new flat detection electrodes were inserted radially closer to the center of the cell, Figure 1 and Figure S1 (Supporting Information). In the NADEL ICR cell evaluated here, the detection electrodes were positioned at $17 \mathrm{~mm}$ radially away from the cell center.

The NADEL as well as a standard Ultra $\left(90^{\circ}\right.$ detection electrodes) ICR cells were mounted, one at a time, onto a hybrid linear ion trap Fourier transform ion cyclotron resonance mass spectrometer (LTQ FT-ICR MS; Thermo Scientific, Bremen, Germany) equipped with a $10 \mathrm{~T}$ actively shielded superconducting magnet (Oxford Nanoscience, Oxon, UK), described elsewhere. Instrument control was performed by the standard electronics and software (Xcalibur, Thermo Scientific). The ions were formed with robotic chip-based nanoelectrospray ionization source (Triversa Nanomate; Advion Biosciences, Ithaca, NY, USA) and transported to the linear

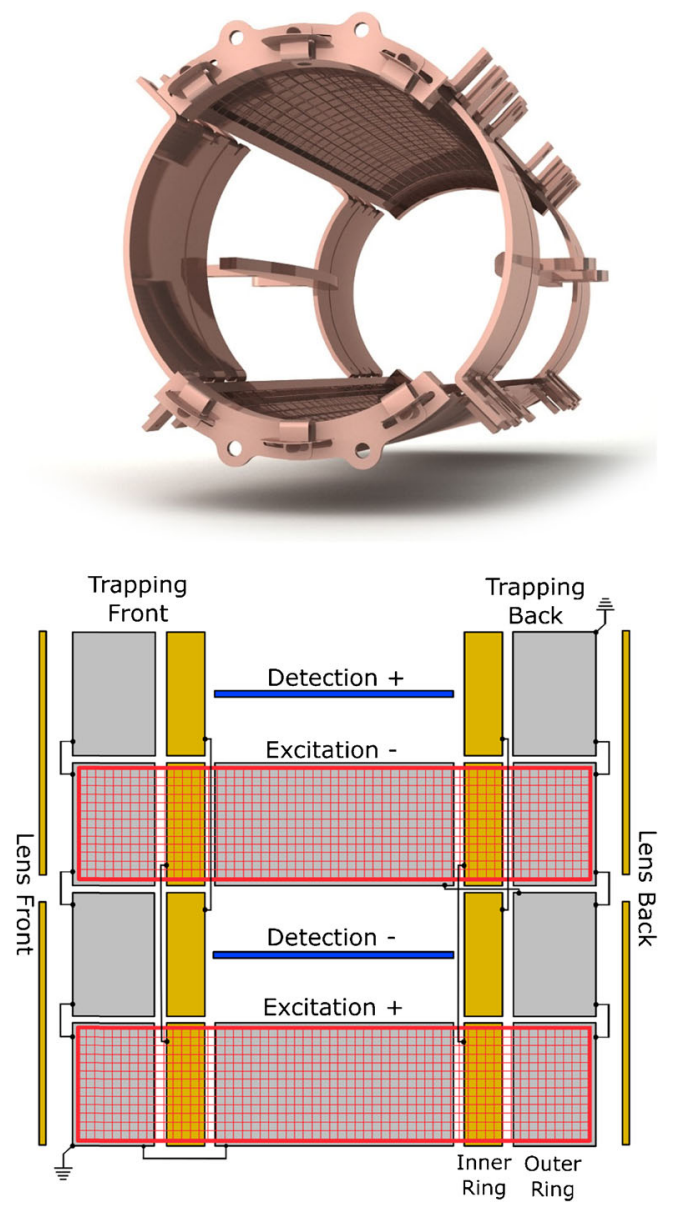

Figure 1. Schematic representation of the narrow aperture detection electrodes (NADEL) ICR cell: (top panel) a 3D view and (bottom panel) its unrolled surface. The NADEL cell contains two pairs, outer and inner, of trapping ring electrodes, excitation grids, conventional $90^{\circ}$ excitation, and narrow aperture (flat) detection electrodes with a thickness of $2 \mathrm{~mm}$ and radius of curvature $R=125 \mathrm{~mm}$ ion trap (LTQ) through an inlet set of multipole ion guides. A predetermined number of charges to be transferred into the ICR cell using another set of multipole ion guides were controlled by the automatic gain control (AGC) function of the LTQ. For petroleomics measurements the AGC function was disabled and accumulation time in the LTQ was set to $50 \mathrm{~ms}$, while the potentials of the inlet transfer system were further optimized manually. For petroleomics analysis, these experimental parameters provided the best correlation of ion distribution envelope (the total width and position of the maximum of ion distribution) between ion detection in LTQ and in the ICR cell. Vacuum conditions in FT-ICR MS with NADEL or Ultra ICR cells were identical (2E-10 Torr).

Ion trapping in both ICR cells was achieved with two pairs of trapping ring electrodes. All sections of outer trapping rings were grounded throughout the complete experimental sequence. A potential of $3 \mathrm{~V}$ was applied to the grid-free sections of inner trapping rings to confine transferred ions inside the ICR cell during ion trapping and relaxation events. The same level of trapping potential was kept during ion excitation event, whereas it was user-defined in the range of $0-10 \mathrm{~V}$ during the ion detection event. Approximately 4.6-fold higher potential was applied to the sections of inner rings covered with grid. The shape of the detection electrodes in NADEL ICR cell was designed to form trapping potential distribution closer to quadratic. For the NADEL ICR cell reported here the distance $r_{0}$ from the detector electrode edge to the ICR cell's axis $z=0$ was selected to be $17 \mathrm{~mm}$, which corresponds to $60 \%$ radius of the original Ultra ICR cell. The shape of the detection electrodes was calculated using the following formula:

$$
r(z)=r_{0}+R\left(1-\sqrt{1-\frac{z^{2}}{R^{2}}}\right)
$$

where $z$ is an axial coordinate and $R$ is the electrode's radius of curvature. For the NADEL ICR cell reported here, with $70 \mathrm{~mm}$ long detection electrodes, we selected a compromise value of $R, 125 \mathrm{~mm}$, as it provides a reasonable ratio of radial and axial trapping electric fields along the $z$-axis (as stated above, trapping potential distribution should be close to quadratic). SIMION calculations confirmed that the selected shape of the detection electrodes provides improved trapping field compared to rectangular narrow aperture detection electrodes, Figures S1 and S2 (Supporting Information). The parameters $r_{0}$ and $R$ can be optimized both numerically (e.g., using SIMION, and experimentally in further studies.

Ion excitation with NADEL ICR cell was technically performed in the same way as with Ultra ICR cell [4]. Coherent motion of ions was excited by standard dipolar frequencysweep excitation in the range of 72-2150 kHz applied during $10 \mathrm{~ms}$ to the excitation grids. The peak-to-peak amplitude of the excitation RF voltage was varied from 0 to $100 \mathrm{~V}$. The particular advantage of the NADEL ICR cell compared with other ICR cell designs, including the Ultra cell, is in the 
improved excitation field distribution, Figure S3, top left (Supporting Information). Due to the positioning of the detection electrodes in the central plane, symmetrically between the top and bottom excitation electrodes, narrow aperture detection electrodes disturb the RF excite field significantly less than the standard $90^{\circ}$ detection electrodes, as employed in Ultra cell. Therefore, ion excitation in the NADEL cell is more efficient than for a regular ICR cell, especially when exciting to a larger radius. Overall, the maximum excitation radius is limited by the location of the detection electrodes. However, to the best of our knowledge, ion excitation to large, $>0.7$ radius, orbits is not employed in modern FT-ICR MS [22, 24, 37]. Moreover, according to the estimation by the authors, most of the ICR cells function with ion excitation to $0.3-0.4$ cell radius because of the increasing of the inhomogeneity of magnetic field at higher radii, although the exact data could not be found in publications. Therefore, the detection electrodes were placed at the corresponding positions, allowing ion excitation to 0.6 cell radius, and thus not reducing the effective ion volume. Note, a finite initial magnetron radius would correspondingly reduce the maximum achievable excitation radius. Figure S3, bottom (Supporting Information) demonstrates the difference in the ion detection conditions between NADEL and Ultra cells. Compared with the standard $90^{\circ}$ detection electrodes, the narrow aperture detection electrodes increase the nonlinear character of ion detection as a function of a postexcitation radius. Importantly, for NADEL cell sensitivity level, detection electrode capacitance of the installed NADEL cell (measured together with the connecting wires and feedthrough) was reduced four-fold compared with the Ultra ICR cell, as expected.

Ion detection was performed using the commercial ion detection configuration of the Ultra ICR cell. Briefly, standard dipolar differential detection was employed to acquire time domain signals (transients). The transients of variable length in the range of $96 \mathrm{~ms}-25 \mathrm{~s}$ were recorded in MIDAS format at 1-5 $\mathrm{MHz}$ sampling frequency (a regime with sinusoidal signals) using the advanced software interface of the built-in data acquisition system (Thermo Scientific). A given number (e.g., 10-100) of transients were averaged to obtain the final transients, which were Hann-apodized and zero-filled once before fast Fourier transformation (FFT) to yield Fourier spectra, which were further transformed to mass spectra using standard data analysis software (Xcalibur; Thermo Scientific) and the pyFTMS framework developed in-house.

Finally, the offset DC potentials in the range from -100 to $+100 \mathrm{mV}$ were applied to the NADEL and Ultra ICR cell detection and excitation electrodes independently relative to each other to vary the position of the ion cloud prior to ion excitation and during ion detection events. The influence of offset potentials on ion motion was monitored with diverse quality attributes of mass spectra (e.g., peak shape and magnetron sidebands [38]). Overall, it was possible to optimize the offset potentials to effectively decrease the magnetron sideband intensities below $1 \%$ relative to the corresponding reduced cyclotron frequency peak for each experiment at the optimal post-excitation ion cyclotron radius.

\section{Results and Discussion}

\section{NADEL ICR Cell Initial Performance Tests: MS and MS/MS Data Acquisition}

A typical broadband mass spectrum obtained with NADEL FT-ICR MS demonstrates efficient simultaneous confinement and detection of ions during $3 \mathrm{~s}$ in a mass range of $\mathrm{m} / \mathrm{z} 300$ 3300 , Figure 2. Similarly, Figure S4 (Supporting Information) shows a broadband mass spectrum of a calibration mixture, revealing simultaneous detection of ions spanning a broad range of $m / z 150-1900$ with acquisition period of $0.384 \mathrm{~s}$. Direct comparison of NADEL FT-ICR mass spectra with those obtained with a linear ion trap (LTQ) on the same instrument demonstrates that for all constituent signals the relative ion abundances are similar (data not shown). The decrease of ion abundance was observed only for $m / z$ less than 300, attributable to expected $\mathrm{m} / \mathrm{z}$-dependent time-of-flight discrimination of ions during their transfer between LTQ and ICR cell [39]. The insets in Figure 2 and Figure S4 (Supporting Information) demonstrate the typical peak shape for monoisotopic peaks of selected compounds.

In-cell tandem mass spectrometry (MS/MS) experiments with NADEL ICR cell demonstrate efficiency comparable to Ultra cell. Typical electron capture dissociation (ECD) [40], infrared multiphoton dissociation (IRMPD) [41], and electron induced dissociation (EID) $[42,43]$ FT-ICR mass spectra are shown in Figure S5 (Supporting Information). MS/MS broadband mass spectra contain analyte peaks with a magnitude spreading over 3-4 decimal orders. Particularly, the spectral dynamic range (ion abundance ratio of the highest to the lowest peaks within a single mass spectrum) [44] of 4.4 orders was achieved for EID MS/MS measurements. Thus, NADEL ICR cell provides dipolar excitation and detection of ions, either injected from an external ion source or produced inside of the cell, in the wide mass range without the discrimination of different $\mathrm{m} / \mathrm{z}$ ions and at a dynamic range comparable to the state-of-the-art FT-ICR MS performance. Note, the employed ion excitation for this mode of operation (generation of sinusoidal ion signals) aims to excite ions to moderate radii, when ions do not yet approach the detection electrodes. The resulting transient components for these regimes are primarily sinusoidal, with a very low $(<5 \%)$ high-order harmonic content.

\section{NADEL ICR Cell Performance: Sensitivity}

To estimate fundamental characteristics of the NADEL ICR cell, in particular the value of magnetron frequency, $\omega_{-}$, and optimal post-excitation ion radius (ion radius at optimized excitation parameters), the reduced cyclotron frequency $\omega_{+}$ and the signal-to-noise ratio (SNR) of peaks corresponding to $\omega_{+}$frequency, its harmonics and interharmonics $\left(n \omega_{+}, n \omega_{+} \pm\right.$ 


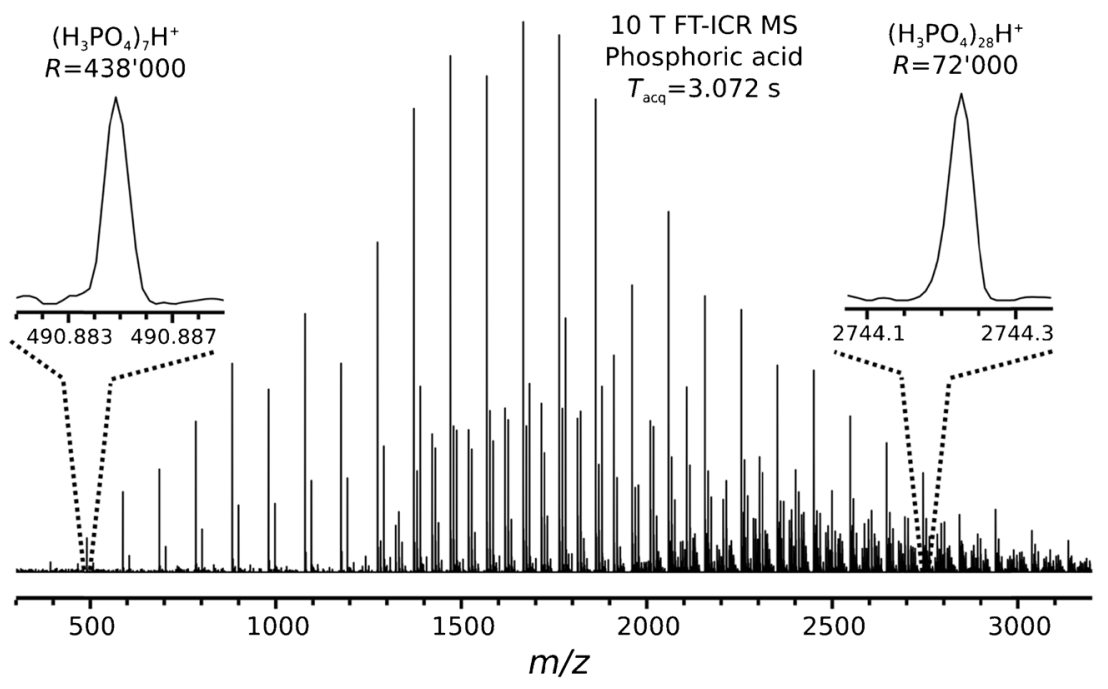

Figure 2. Broadband mass spectrum of a polyphosphoric acid sample, acquired with 10 T FT-ICR MS equipped with NADEL ICR cell. The mass spectrum was obtained from a sum of 30 transients recorded in the mass range of $300-3300 \mathrm{~m} / \mathrm{z}$ with the acquisition period $3.072 \mathrm{~s}$ and number of charges $1 \mathrm{E} 6$ (AGC)

$k \omega_{\text {- }}$, where $\left.n, k=1,2,3\right)$ were measured as functions of excitation energy for isolated singly protonated ions of MRFA $(\mathrm{m} / \mathrm{z} 524.3)$ at different trapping potentials applied during ion detection event, Figure 3. As expected, the SNR of the peak corresponding to the reduced cyclotron frequency $\omega_{+}$linearly increases with excitation amplitude (cyclotron radius).
However, a significant decrease of the SNR was observed for excitation amplitudes higher than approximately $40 \mathrm{~V}$. On the other hand, the magnitude ratio of the harmonic $3 \omega_{+}$to the $\omega_{+}$ peak increased with the excitation amplitude, Figure 3 top left, meaning that the post-excitation cyclotron radius continues to increase with the corresponding growth of the high-order
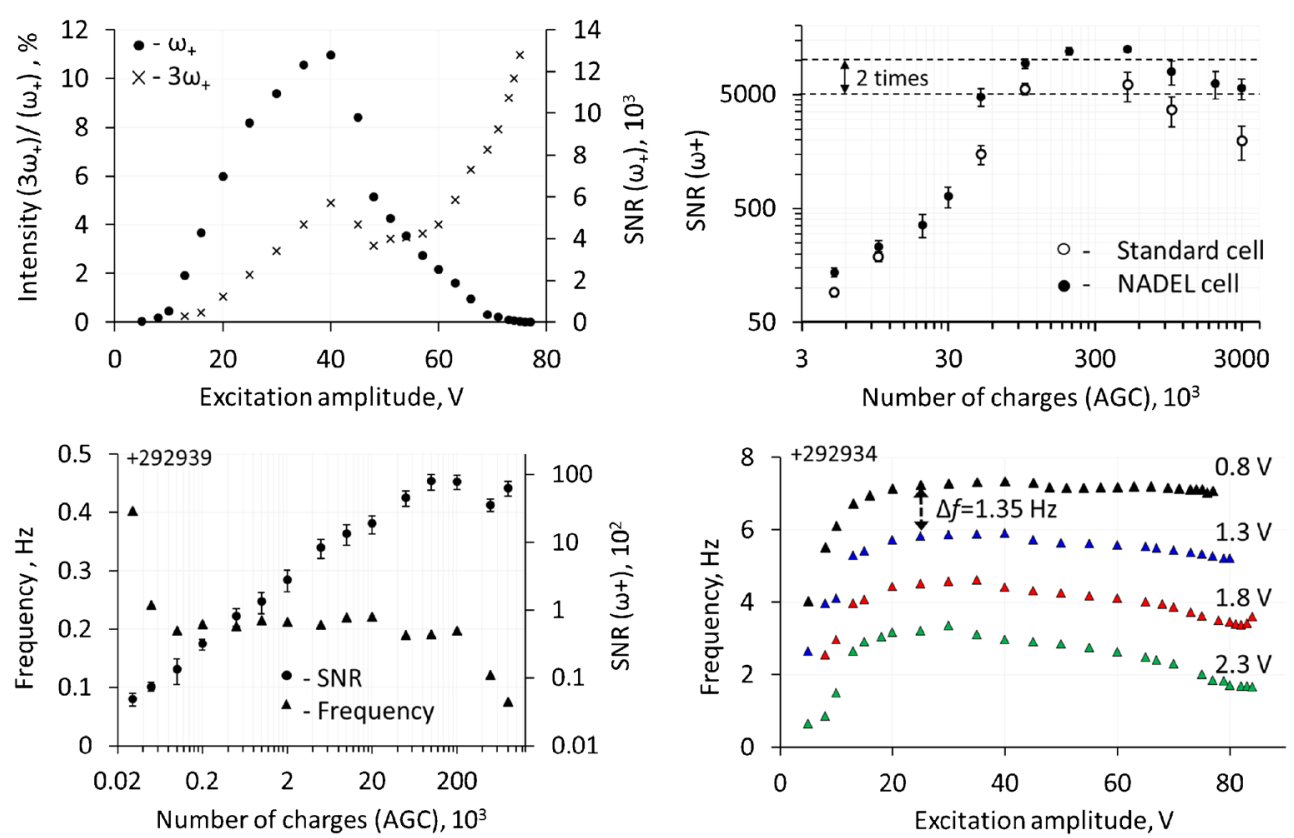

Figure 3. Characterization of the NADEL ICR cell in 10 T FT-ICR MS. (Top left): relative intensity of the harmonic $3 \omega_{+}$(w.r.t. the peak $\left.\omega_{+}\right)$and signal-to-noise ratio (SNR), as absolute intensity w.r.t. the mean of baseline noise, of the peak $\omega_{+}$, as a function of the excitation amplitude. (Top right) dependencies of the SNR of the peak $\omega_{+}$on the defined number of charges (logarithmic scales) were obtained under identical instrumental parameters for both the standard (open circles) and NADEL (solid circles) ICR cells. (Bottom left) dependencies of the frequency (triangles) and the SNR value (circles) of the peak $\omega_{+}$on the user-defined value of number of charges (AGC) in the wide range 2E1-2E6. (Bottom right) dependence of the frequency of the peak $\omega_{+}$on the excitation amplitude for different trapping potentials. All experimental data were acquired for isolated singly protonated MRFA peptide $(\mathrm{m} / \mathrm{z} 524.3)$ with the acquisition period of $3.072 \mathrm{~s}$ and $10 \mathrm{~ms}$ of excitation duration 
harmonics. The SNR decline for the fundamental frequency, $\omega_{+}$, is presumably due to the reduced harmonicity of the trapping electric potential and homogeneity of the excitation electric field for high excitation amplitudes, which cause phase decoherence of ion clouds, leading to a decrease of ion signal amplitude. Additionally, even harmonics of $\omega_{+}$were observed in the mass spectra. The presence of even harmonics testifies for a non-zero magnetron radius and an initial radial shift of an ion cloud from the axis of the ICR cell. Notably, even harmonics' magnitudes were reduced to a level less than $2 \%$ relative to the $\omega_{+}$peak via fine tuning of the offset DC potentials applied to the excitation and detection electrodes independently for each trapping potential.

The sensitivity levels of the NADEL ICR cell and the standard Ultra cell with $90^{\circ}$ detection electrodes were compared for an isolated singly protonated MRFA peptide $(\mathrm{m} / \mathrm{z}$ 524.3) under identical optimal instrumental parameters for both cells. As expected, a signal-to-noise ratio (SNR) of peak corresponding to the reduced cyclotron frequency $\omega_{+}$linearly increased with the number of charges (ion abundance) for both NADEL and Ultra ICR cells, Figure 3 top right. The SNR values reached a plateau at around 1E5-2E5 number of charges in the isolation mode for both cells as well. This is likely due to the limitations of accumulation in LTQ and ion transport from LTQ to ICR cell for ions with the same nominal mass-to-charge ratio. Importantly, Figure 3 top right shows the increase of the peak SNR almost two times for the NADEL cell compared with the standard Ultra cell. Therefore, the experimental results demonstrate higher signal voltage developed across the detection circuit of the NADEL ICR cell compared with the corresponding signal in the standard $90^{\circ}$ electrode Ultra ICR cell, despite the significantly reduced surface area of detection electrodes of the NADEL cell. This result can be explained by the substantially lower capacitance of the flat detect electrodes and shorter distance between the orbit of excited ions and detection electrodes. It is thus not surprising that only a few charges, approximately 10 ions $(\mathrm{AGC}=30)$ per cell load, of triply protonated substance $\mathrm{P}(\mathrm{m} / \mathrm{z} 449.9)$ ions were detected with acquisition period of $384 \mathrm{~ms}$ and five transients averaging, Figure S6 (Supporting Information). This result correlates well with the known sensitivity performance level of 50 charges of the state-of-the-art FT-ICR MS with standard ICR cells.

\section{NADEL ICR Cell Performance: Mass Accuracy}

For low trapping potentials $(<1 \mathrm{~V})$, the reduced cyclotron frequency $\omega_{+}$peak remained within the frequency range of $1 \mathrm{ppm}$ over the entire range of excitation amplitudes where ion signal can be detected, except the region of low excitation amplitudes, Figure 3, bottom right. However, frequency reduced with an increase in the trapping potential for high excitation amplitudes due to reduced harmonicity of the trapping electric potential. Thus, the electric field distribution of the NADEL ICR cell is uniform in the wide range of excitation amplitudes (post-excitation radii) at low trapping potentials. Note, the DC offset potentials were adjusted for each trapping potential independently when constructing these dependencies. Furthermore, at low excitation amplitudes (small postexcitation radii) for all trapping potentials a space charge of significant ion abundance $(\mathrm{AGC}=5 \mathrm{E} 4)$ is the reason for the observed frequency shift, as reported earlier [45]. A scaling factor of the magnetron frequency was experimentally determined to be $2.7 \mathrm{~Hz} / \mathrm{V}$, Figure 3, bottom right. Experimental results allowed us to define optimal trapping potentials $0.8-1.0$ $\mathrm{V}$ and excitation amplitude of 30-40 V with a duration of 10 ms. Additionally, the stability of the reduced cyclotron frequency measured for isolated singly protonated ions of MRFA $524.3 \mathrm{~m} / \mathrm{z}$ with trapping potential of $1.0 \mathrm{~V}$ and excitation amplitude of $30 \mathrm{~V}$ indicates the absence of space charge effect in the wide range of number of charges, $\mathrm{AGC}=1 \mathrm{E} 2-1 \mathrm{E} 5$, for the NADEL ICR cell, Figure 3, bottom left.

Mass calibration of the FT-ICR mass spectrometer equipped with the NADEL ICR cell was performed via the standard two parametric calibration function using nine monoisotopic ions of ESI-L low concentration tuning mixture (Agilent). The mass calibration function was determined using two AGC values (5E5 and 2E6) in the mass range of $m / z$ 300-1400 (four calibrants) with acquisition period of $0.568 \mathrm{~s}$ and trapping potentials of 1 and $2 \mathrm{~V}$, as well as in the range of $\mathrm{m} / \mathrm{z} 300$ 3000 (nine calibrants) with acquisition period of $1.336 \mathrm{~s}$ and trapping potential of $1 \mathrm{~V}$ resulting in six calibration parameter sets, Table 1.

Mass calibration was evaluated by considering the mass measurement accuracies achieved in the same mass ranges for analyte peaks of polyphosphoric acid $\mathrm{H}_{\mathrm{n}+2} \mathrm{P}_{\mathrm{n}} \mathrm{O}_{3 \mathrm{n}+1}, n=3-$ 30 , (monoisotopic peaks of protonated and ammonium adducts, as well as the ${ }^{18} \mathrm{O}$ isotopologue of the protonated species), Table 1. Only analyte peaks with SNR higher than 3 were taken into account. The mass error did not exceed $750 \mathrm{ppb}$ level with the root-mean-square (RMS) value of $330 \mathrm{ppb}$ in the mass range 300-1400 m/z for both AGC settings and both trapping potentials. These results are comparable to those obtained using conventional ICR cells with external mass calibration [24, 46, 47]. Mass error increased for wider mass range, especially for

Table 1. RMS Mass Accuracy of Agilent ESI-L Low Concentration Tuning Mixture (Internal Calibration) and Polyphosphoric Acid (External Calibration, Based on the ESI-L Mixture) Obtained with Two Values of a Number of Charges (AGC Equal to 5E5 or 2E6)

\begin{tabular}{|c|c|c|c|c|}
\hline \multirow[t]{3}{*}{ AGC } & \multicolumn{4}{|c|}{ RMS mass error, ppm } \\
\hline & \multicolumn{2}{|c|}{$\begin{array}{l}\text { Mass range: } 300-1400 \mathrm{~m} / z \\
T_{\mathrm{acq}}=568 \mathrm{~ms} ; V_{\mathrm{trap}}=1 \mathrm{~V}(2 \mathrm{~V})\end{array}$} & \multicolumn{2}{|c|}{$\begin{array}{l}\text { Mass range: } 300-3000 \mathrm{~m} / \mathrm{z} \text {; } \\
T_{\text {acq }}=1336 \mathrm{~ms} ; V_{\text {trap }}=1 \mathrm{~V}\end{array}$} \\
\hline & Internal & External & Internal & External \\
\hline $5 \mathrm{E} 5$ & $0.06(0.14)$ & $0.33(0.24)$ & 0.47 & 0.65 \\
\hline 2E6 & $0.19(0.04)$ & $0.23(0.20)$ & 1.46 & 1.64 \\
\hline
\end{tabular}

Mass calibration procedures were performed in the mass range of $\mathrm{m} / \mathrm{z} 300-1400$ with acquisition period of $0.568 \mathrm{~s}$ and $m / z 300-3000$ with acquisition period of $1336 \mathrm{~ms}$, using 30 summed transients for each of the ranges. The corresponding dependencies for external calibration are shown in Figure S7 (Supporting Information) 
higher AGC value; the corresponding dependencies are given in Figure S7 (Supporting information).

To rationalize these results, the effect of space charge on mass accuracy was investigated using polyphosphoric acid with internal calibration. Specifically, mass calibration was performed for three mass ranges and varied AGC values (3E5-6E6) with acquisition period of $1336 \mathrm{~ms}$, Table 2. Figure S7 (Supporting Information) demonstrates the SNR values of analyte peaks linearly increasing with an increase of the AGC value in the current range. The RMS mass accuracy of $60 \mathrm{ppb}$ was achieved for eight monoisotopic peaks of polyphosphoric acid in the range of $300-1000 \mathrm{~m} / \mathrm{z}$ and $\mathrm{AGC}=7 \mathrm{E} 5$. Furthermore, the RMS mass error remained within $200 \mathrm{ppb}$ for the number of charges up to $\mathrm{AGC}=2 \mathrm{E} 6$ for low mass calibration range up to $1000 \mathrm{~m} / z$, Table 2 and Figure S7 (Supporting Information). These results are comparable to those obtained with conventional and harmonized ICR cells for internal calibration [24, 46, 48]. The mass error became more pronounced for higher AGC values, 3E6, and more as can be expected due to an increased space-charge field [45, 49], especially for the highest $\mathrm{m} / \mathrm{z}$ peaks due to higher analyte density per nominal mass in that $\mathrm{m} / \mathrm{z}$ region, Figure 2 and Figure S7 (Supporting Information).

Thus, mass accuracy performance with NADEL ICR cell is comparable to those obtained with conventional and harmonized ICR cells. This conclusion also follows from the analysis of a crude oil fraction using NADEL ICR cell, vide infra. Application of absorption mode FT signal processing allows improving RMS mass accuracy values two times, as expected [50-52]. However, the observed increase in mass inaccuracy when a very wide $m / z$ range is analyzed, Figure S7 (Supporting Information) and Table 1, needs further investigation. Particularly, influence of the modified trapping field should be considered. Indeed, Figure S1 (Supporting Information) shows that the detection electrode configuration described here introduces an azimuthal component of a trapping electric field, previously avoided in FT-ICR MS cell designs. On the other hand, it is known that the offset DC potentials typically employed to reduce harmonic content in FT-ICR mass spectra and improve peak shapes may introduce a similar azimuthal component to the trapping field distribution. Therefore, the exact character, destructive or constructive, of the modified trapping field is to be evaluated. Finally, further optimization of narrow aperture detection electrodes may reduce the introduced modulation of the trapping electric field.

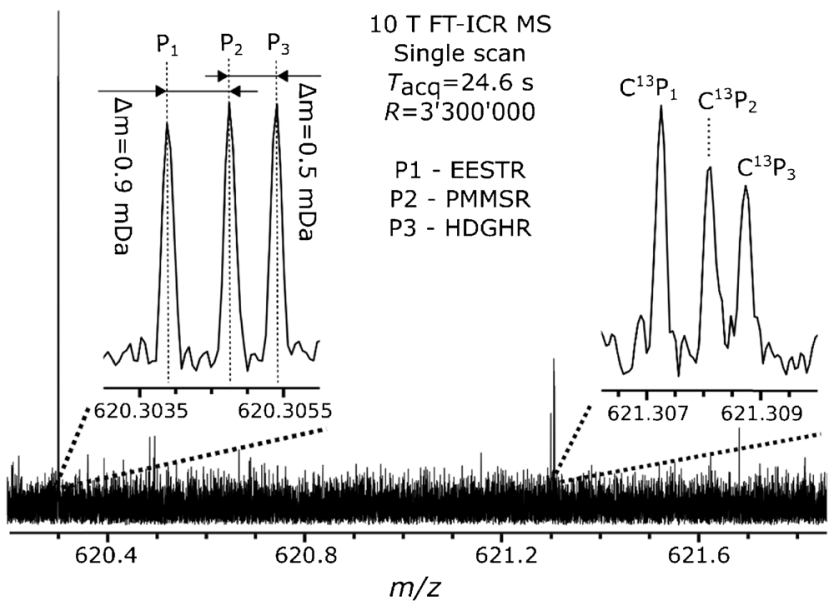

Figure 4. Isobaric peptide mixture measurements with NADEL cell in $10 \mathrm{~T}$ FT-ICR MS. The resolving power 3'300'000 was achieved for a single scan with acquisition period of $24.576 \mathrm{~s}$ and number of charges (AGC) of 30'000 for isolated ions of singly protonated peptides $\mathrm{P} 1, \mathrm{P} 2$, and P3

\section{NADEL ICR Cell Performance: Resolving Power}

The resolving power performance of the NADEL ICR cell was evaluated in the analysis of peptide and protein samples with maximum detection period of $24.576 \mathrm{~s}$ for the employed hardware configuration. Acquisition of longer transients was not possible because of the technical limitations imposed by the on-board LTQ FT computer memory, whereas the lifetime of excited and coherent ion motion apparently surpasses this temporal limitation; for example see Figure S8 (Supporting Information). Notably, the achieved ion detection period of $\sim 25 \mathrm{~s}$ is the record for LTQ FTMS instruments reports.

Figure 4 shows a mass spectrum of three isobaric singly protonated peptides $\mathrm{P} 1, \mathrm{P} 2$, and $\mathrm{P} 3$ (nominal $\mathrm{m} / \mathrm{z}$ of monoisotopic peaks is 620) with mass differences of 0.9 and $0.5 \mathrm{mDa}$ between the pairs of peptides. Notably, mass of $0.5 \mathrm{mDa}$ is close to the mass of an electron. Expanded segments of the mass spectrum show baseline-resolved monoisotopic and ${ }^{13} \mathrm{C}$ peaks for the three peptides of interest with the resolving power reaching $3^{\prime} 300^{\prime} 000$ for a single scan. To avoid the peak coalescence effect, a relatively low number of ions were employed (AGC setting of 3E4). The particular importance of the result reported in Figure 4 is in the long time of ion cloud coherence after excitation, which allows recording of $25 \mathrm{~s}$ and (potentially) longer transients. The reported separation of this

Table 2. RMS Mass Accuracy of Polyphosphoric Acid (Internal Calibration) as a Function of Number of Charges

\begin{tabular}{|c|c|c|c|c|c|}
\hline \multirow[t]{2}{*}{ Mass range, $m / z$} & \multicolumn{5}{|c|}{ RMS mass error, ppm } \\
\hline & $\mathrm{AGC}=3 \mathrm{E} 5$ & $\mathrm{AGC}=7 \mathrm{E} 5$ & $\mathrm{AGC}=2 \mathrm{E} 6$ & $\mathrm{AGC}=4 \mathrm{E} 6$ & $\mathrm{AGC}=6 \mathrm{E} 6$ \\
\hline 300-1000 (8 peaks) & 0.11 & 0.06 & 0.18 & 0.37 & 0.57 \\
\hline $300-2000$ (19 peaks) & 0.69 & 0.44 & 0.98 & 1.67 & 2.21 \\
\hline $300-3100$ (29 peaks) & 0.92 & 0.88 & 1.43 & 2.54 & 3.35 \\
\hline
\end{tabular}

Mass calibration was performed in the mass ranges of $m / z 300-1000,300-2000$, and 300-3100. For each of the ranges, 50 transients with acquisition period of $1336 \mathrm{~ms}$ were summed. The corresponding dependence is shown in Figure S7 (Supporting Information) 
peptide triplet can be compared with the notable example of a peptide doublet separation (mass difference close to the mass of an electron) by Marshall and co-workers on a 9.4 T FT-ICR MS [53].

Another notable example of resolving power performance provided by NADEL cell for peptide and protein analysis is the acquisition of the isotopic fine structure distribution of triply protonated substance $\mathrm{P}(449.9 \mathrm{~m} / \mathrm{z})$ and major isotopologues of $11+$ charge state of bovine ubiquitin $(779.0 \mathrm{~m} / \mathrm{z})$, Figure 5 . The mass spectrum of substance $\mathrm{P}$ was obtained for 10 summed transient signals; the summed signal is shown in Figure S8 (Supporting Information). Mass spectrum of bovine ubiquitin was obtained from 20 summed single transients. Isotopic fine structure components of substance $\mathrm{P}$ and bovine ubiquitin are resolved with resolution of $4^{\prime} 400^{\prime} 000$ and $2^{\prime} 900^{\prime} 000$, respectively.

To evaluate NADEL ICR cell performance for heavy protein analysis, protein mass measurements were performed for a bovine serum albumin (BSA) with direct infusion ESI.
Broadband mass spectrum of BSA obtained by averaging of 50 single scans of $1.536 \mathrm{~s}$ shows the charge state distribution in the range from $35+$ up to $72+$, Figure S9 (Supporting Information). Furthermore, the resolving power of $320^{\prime} 000$ was achieved for an isolated $\mathrm{BSA}^{48+}$ charge state with a single scan and acquisition period of 6.144 s, Figure S10 (Supporting Information).

Finally, a petroleomic sample was chosen to evaluate the NADEL ICR cell performance for complex mixture analysis. A positive ESI broadband mass spectrum of a fraction of South American crude oil was acquired with an acquisition period of $3.072 \mathrm{~s}$, Figure 6 . The insets demonstrate mass scale expansions at $451 \mathrm{~m} / \mathrm{z}$ nominal mass, revealing typical representative spectral compositions for a petroleum sample with resolving power of 450'000. Mass assignments are given in Table 3. Additionally, mass accuracy values for the 2715 monoisotopic peaks identified in the broadband mass spectrum of a crude oil fraction in the mass range of 300-700 m/z were measured as a
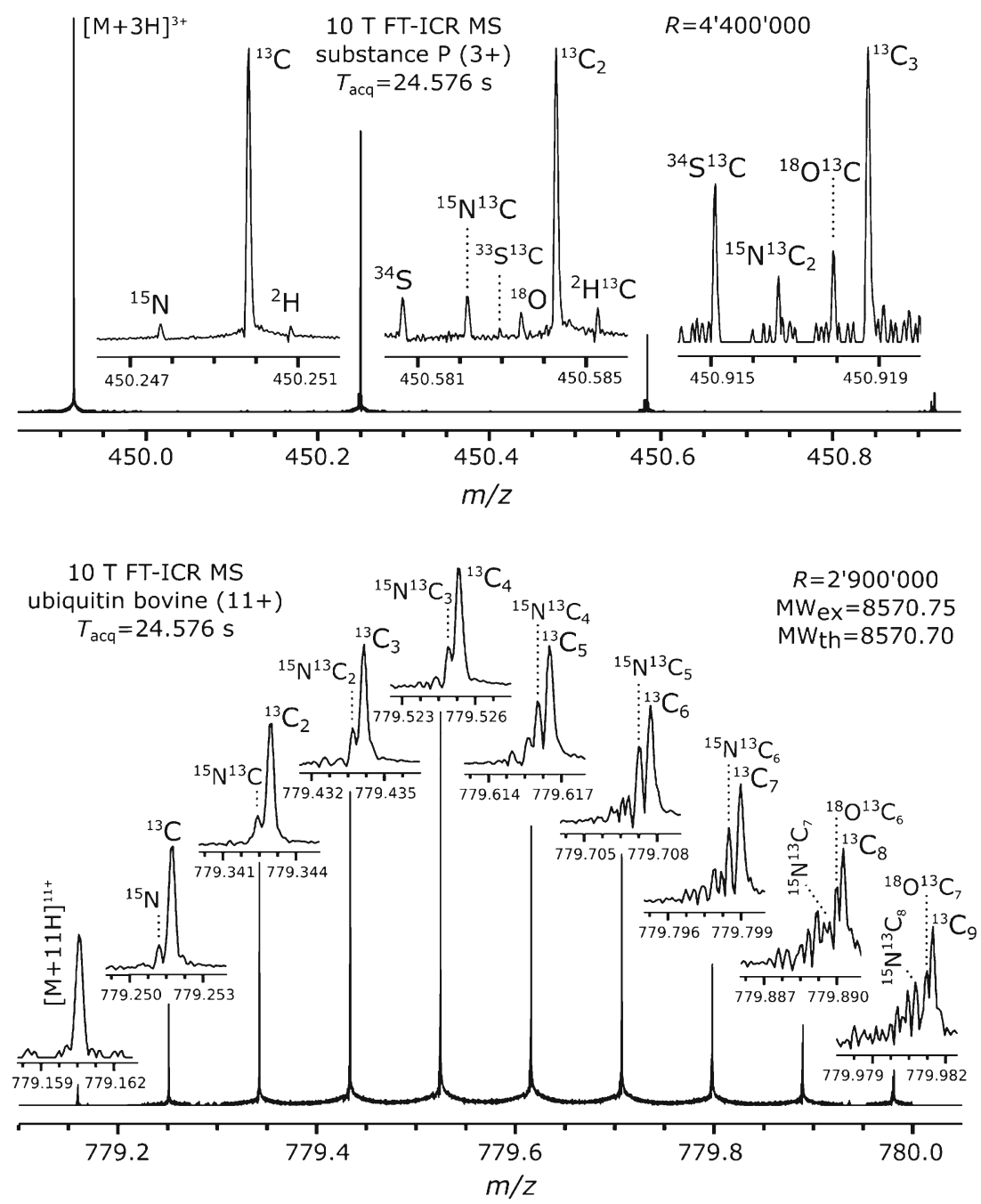

Figure 5. Peptide (substance P, top panel) and protein (ubiquitin, bottom panel) isotopic fine structure measurements with NADEL cell in 10 T FT-ICR MS. Mass spectrum of isolated triply protonated substance P $449.9 \mathrm{~m} / \mathrm{z}$ was obtained from a sum of 10 transients of 24.576 s each. The corresponding transient is shown in Figure S8 (Supporting Information). Mass spectrum of bovine ubiquitin was obtained from sum of 20 transients of $24.576 \mathrm{~s}$ acquired for isolated 11+ ions 


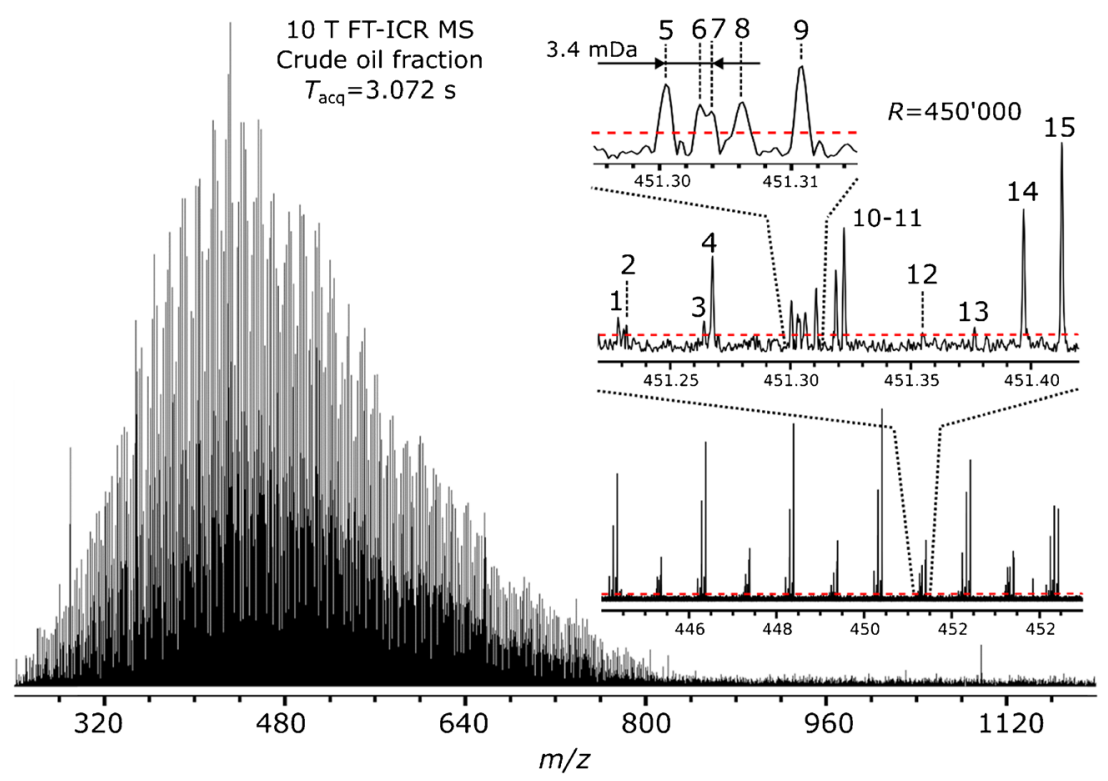

Figure 6. ESI broadband mass spectrum of a crude oil fraction obtained with NADEL ICR cell in 10 T FT-ICR MS from the averaging of 600 transients of $3.072 \mathrm{~s}$ each in the positive-ion mode. Insets show expanded regions around (top) $451 \mathrm{~m} / z$ and (bottom) 445-453 m/z for the displayed broadband mass spectrum. Only peaks above a certain threshold (dotted red line) were considered. Annotated peaks are listed in Table 3

function of $\mathrm{m} / \mathrm{z}$ and SNR by using standard two-parametric equation and internal calibration based on 29 monoisotopic peaks of a prominent homologous series, Figure S11 (Supporting information). Overall, 5142 peaks were detected with the intensity higher than $2 \%$ relative to the highest magnitude peak in mass spectrum. RMS mass accuracy and mean values were $236 \mathrm{ppb}$ and $0.7 \mathrm{ppb}$, respectively. As expected, mass error increases with decreasing SNR and increasing $\mathrm{m} / \mathrm{z}$ value.

To conclude, the combined benefits of trapping and excitation field configuration in the NADEL cell provide comparable performance to standard ICR cells in routine analysis in regard to resolving power performance. The obtained characteristics approach those of the most sophisticated ICR cells with complex approaches to electric field harmonization, either statically or dynamically, for the investigated $10 \mathrm{~T}$ magnetic field [24, $29,46]$. The described NADEL ICR cell has been employed to perform routine mass analysis in our laboratory and demonstrated an ability to achieve the required analytical objectives of molecular structural analysis in a number of applications, for example in the analysis of supramolecular complexes [54].

\section{NADEL ICR Cell Concept Advantages}

The particular benefits of the NADEL ICR cell concept for current and envisioned FT-ICR MS configurations include: (1) enhanced dipolar ion excitation conditions because of improved configuration of excite electric field. The latter is achieved by reduced excitation field disturbance by radially inserted detection electrodes compared with the standard $90^{\circ}$

Table 3. Peak Assignments and Mass Errors in the Segments Around $451 \mathrm{~m} / \mathrm{z}$ of the Positive ESI Broadband Mass Spectrum of a Crude Oil fraction. Corresponding Inset is Shown in Figure 6

\begin{tabular}{|c|c|c|c|c|}
\hline Peak no. & Composition & $m / z$ & $\Delta m / m, \mathrm{ppm}$ & Heteroatom class \\
\hline 1 & ${ }^{13} \mathrm{C}_{1}{ }^{12} \mathrm{C}_{30} \mathrm{H}_{32} \mathrm{NS}$ & 451.228355 & -0.135 & NS \\
\hline 2 & ${ }^{13} \mathrm{C}_{1}{ }^{12} \mathrm{C}_{27} \mathrm{H}_{36} \mathrm{NS}_{2}$ & 451.231726 & -0.098 & $\mathrm{NS}_{2}$ \\
\hline 3 & $\mathrm{C}_{27} \mathrm{H}_{40} \mathrm{O}_{2} \mathrm{SNa}$ & 451.264122 & 0.119 & $\mathrm{O}_{2} \mathrm{~S}$ \\
\hline 4 & $\mathrm{C}_{24} \mathrm{H}_{44} \mathrm{O}_{2} \mathrm{~S}_{2} \mathrm{Na}$ & 451.267496 & 0.059 & $\mathrm{O}_{2} \mathrm{~S}_{2}$ \\
\hline 5 & $\mathrm{C}_{28} \mathrm{H}_{44} \mathrm{OSNa}$ & 451.300510 & -0.069 & OS \\
\hline 6 & $\mathrm{C}_{30} \mathrm{H}_{43} \mathrm{OS}$ & 451.302913 & 0.295 & OS \\
\hline 7 & $\mathrm{C}_{25} \mathrm{H}_{48} \mathrm{OS}_{2} \mathrm{Na}$ & 451.303879 & -0.337 & $\mathrm{OS}_{2}$ \\
\hline 8 & $\mathrm{C}_{27} \mathrm{H}_{47} \mathrm{OS}_{2}$ & 451.306285 & 0.120 & $\mathrm{OS}_{2}$ \\
\hline 9 & $\mathrm{C}_{32} \mathrm{H}_{39} \mathrm{~N}_{2}$ & 451.310778 & -0.152 & $\mathrm{~N}_{2}$ \\
\hline 10 & ${ }^{13} \mathrm{C}_{1}{ }^{12} \mathrm{C}_{32} \mathrm{H}_{40} \mathrm{~N}$ & 451.318881 & -0.209 & $\mathrm{~N}$ \\
\hline 11 & ${ }^{13} \mathrm{C}_{1}{ }^{12} \mathrm{C}_{29} \mathrm{H}_{44} \mathrm{NS}$ & 451.322255 & -0.035 & NS \\
\hline 12 & $\mathrm{C}_{29} \mathrm{H}_{48} \mathrm{O}_{2} \mathrm{Na}$ & 451.354651 & 0.202 & $\mathrm{O}_{2}$ \\
\hline 13 & $\mathrm{C}_{26} \mathrm{H}_{53} \mathrm{NOSNa}$ & 451.375783 & 0.078 & NOS \\
\hline 14 & $\mathrm{C}_{29} \mathrm{H}_{55} \mathrm{OS}$ & 451.396816 & 0.155 & OS \\
\hline 15 & ${ }^{13} \mathrm{C}_{1}{ }^{12} \mathrm{C}_{31} \mathrm{H}_{52} \mathrm{~N}$ & 451.412784 & 0.034 & $\mathrm{~N}$ \\
\hline
\end{tabular}


degrees wide detection electrodes, Figure S3 (Supporting Information). Further improvement of excite electric field distribution can be presumably obtained by utilization of wide aperture excitation electrodes with an optimized angle, which can be now varied up to $180^{\circ}$. Interestingly, flat (wide aperture) excitation electrodes (capacitor configuration) may be considered to provide an optimum excitation field configuration; (2) simultaneous detection of a number of transients or detection at frequency multiples when several NADEL pairs are employed, for instance leading to an optimized design of the recently implemented quadruple frequency multiple detection ICR cell [36]; (3) enabling implementation of efficient quadrature ion excitation and quadrupolar ion detection schemes when two pairs of $90^{\circ}$ wide excite electrodes and two NADEL pairs are employed without the need for a high-frequency switch between excitation and detection modes. Quadrupolar ion detection should further benefit high mass accuracy and high dynamic range demanding applications by providing measurements of trapping potential-insensitive unperturbed ion cyclotron frequency $[23,55]$; (4) creation of special ion motion conditions in ICR cell even with dipolar ion excitation leading to ion detection at unperturbed cyclotron frequency [56]; (5) facilitating and improving the efficiency of fluorescence-based ion spectroscopy due to substantially increased optical access to and from the cell [57-59]; and, last but not least, (6) enabling high throughput high-resolution mass spectrometry by accelerating ions to high post-excitation radii when, significantly, an order of magnitude higher resolving power can be obtained in the same ion detection period. The latter regime requires the development of the matching signal processing methods, capable of efficient analysis of periodic non-sinusoidal transients with high order harmonics [60].

\section{Conclusions}

We designed and implemented an ICR cell with a pair of narrow aperture detection electrodes (NADEL) for improving the analytical capabilities and increasing the mass analyzer design flexibility of FT-ICR MS. The obtained results demonstrate that the narrow aperture (flat) detection electrodes provide comparable or superior performance relative to the standard (commercial) ICR cells in modern FT-ICR MS. As proven here, the ability to replace the currently employed wide aperture (azimuthally large) detection electrodes in all ICR cell designs with the narrow aperture ones opens new avenues for ICR cell development and FT-ICR MS capabilities. A number of advantages and envisioned benefits validate the importance of the described ICR cell development. Rationalizing the underlying ion physics phenomena leading to these capabilities (e.g., unperturbed ion cyclotron frequency detection with dipolar ion detection) requires in-depth theoretical analysis of the described NADEL ICR cell and its further modifications.

\section{Acknowledgments}

The authors are grateful to Konstantin O. Zhurov for technical assistance and discussions. The authors also express their gratitude to Thermo Fisher Scientific Inc. for providing access under license to LTQ FT transient signals and advanced user interface. They acknowledge financial support through the Swiss National Science Foundation (SNF project 200021125147/1) and the European Research Council (ERC Starting Grant 280271).

\section{References}

1. Marshall, A.G., Hendrickson, C.L., Jackson, G.S.: Fourier transform ion cyclotron resonance mass spectrometry: a primer. Mass Spectrom. Rev. 17, 1-35 (1998)

2. Amster, I.J.: Fourier transform mass spectrometry. J. Mass Spectrom. 31, 1325-1337 (1996)

3. Marshall, A.G., Hendrickson, C.L.: High-resolution mass spectrometers. In: Annual Review of Analitical Chemistry, vol. 1, pp. 579-599 (2008)

4. Scigelova, M., Hornshaw, M., Giannakopulos, A., Makarov, A.: Fourier transform mass spectrometry. Mol. Cell. Proteomics 10, M111.009431 (2011)

5. Xian, F., Hendrickson, C.L., Marshall, A.G.: High resolution mass spectrometry. Anal. Chem. 84, 708-719 (2012)

6. Tsybin, Y.O.: Structural analysis of complex molecular systems by highresolution and tandem mass spectrometry. In: Pignataro, B. (eds.) Discovering the future of molecular sciences, Wiley-VCH Verlag GmbH \& Co. KGaA, pp. 63-91(2014)

7. Hsu, C.-S., Hendrickson, C.L., Rodgers, R.P., McKenna, A.M., Marshall, A.G.: Petroleomics: advanced molecular probe for petroleum heavy ends. J. Mass Spectrom. 46, 337-343 (2011)

8. Zubarev, R.A., Makarov, A.: Orbitrap mass spectrometry. Anal. Chem. 85, 5288-5296 (2013)

9. Zhurov, K.O., Kozhinov, A.N., Tsybin, Y.O.: Evaluation of high-field Orbitrap Fourier transform mass spectrometer for petroleomics. Energy Fuel 27, 2974-2983 (2013)

10. Fornelli, L., Damoc, E., Thomas, P.M., Kelleher, N.L., Aizikov, K., Denisov, E., Makarov, A., Tsybin, Y.O.: Analysis of intact monoclonal antibody IgG1 by electron transfer dissociation Orbitrap FTMS. Mol. Cell. Proteomics 11, 1758-1767 (2012)

11. Tsybin, Y.O., Fornelli, L., Stoermer, C., Luebeck, M., Parra, J., Nallet, S., Wurm, F.M., Hartmer, R.: Structural analysis of intact monoclonal antibodies by electron transfer dissociation mass spectrometry. Anal. Chem. 83, 8919-8927 (2011)

12. Marshall, A.G., Hendrickson, C.L.: Fourier transform ion cyclotron resonance detection: principles and experimental configurations. Int. J. Mass Spectrom. 215, 59-75 (2002)

13. Miladinovic, S.M., Kozhinov, A.N., Gorshkov, M.V., Tsybin, Y.O.: On the utility of isotopic fine structure mass spectrometry in protein identification. Anal. Chem. 84, 4042-4051 (2012)

14. Hebert, A.S., Merrill, A.E., Bailey, D.J., Still, A.J., Westphall, M.S., Strieter, E.R., Pagliarini, D.J., Coon, J.J.: Neutron-encoded mass signatures for multiplexed proteome quantification. Nat. Methods 10, 332-334 (2013)

15. McAlister, G.C., Huttlin, E.L., Haas, W., Ting, L., Jedrychowski, M.P., Rogers, J.C., Kuhn, K., Pike, I., Grothe, R.A., Blethrow, J.D., Gygi, S.P.: Increasing the multiplexing capacity of TMTs using reporter ion isotopologues with isobaric masses. Anal. Chem. 84, 7469-7478 (2012)

16. Nikolaev, E.N., Kostyukevich, Y.I., Vladimirov, G.N.: Fourier transform ion cyclotron resonance (FT ICR) mass spectrometry: theory and simulations. Mass Spectrom. Rev. doi:10.1002/mas.21422

17. Marshall, A.G.: Milestones in Fourier transform ion cyclotron resonance mass spectrometry technique development. Int. J. Mass Spectrom. 200, 331-356 (2000)

18. Guan, S., Marshall, A.G.: Ion traps for Fourier transform ion cyclotron resonance mass spectrometry: principles and design of geometric and electric configurations. Int. J. Mass Spectrom. Ion Processes 146/147, 261-296 (1995)

19. Kaiser, N., Weisbrod, C., Quinn, J., Blakney, G.T., Beu, S., Chen, T., Henrickson, C. L., Marshall, A.G.: Development of an FT-ICR mass spectrometer in preparation for 21 tesla. Proceedings of the 62nd American 
Society for Mass Spectrometry Conference on Mass Spectrometry and Allied Topics, Baltimore, MD, June 15-19 (2014)

20. Guan, S., Gorshkov, M.V., Marshall, A.G.: Circularly polarized quadrature excitation for Fourier-transform ion cyclotron resonance mass spectrometry. Chem. Phys. Lett. 198, 143-148 (1992)

21. Guan, S., Wahl, M.C., Marshall, A.G.: Elimination of frequency drift from Fourier transform ion cyclotron resonance mass spectra by digital quadrature heterodyning: ultrahigh mass resolving power for laser-desorbed molecules. Anal. Chem. 65, 3647-3653 (1993)

22. Schweikhard, L., Marshall, A.G.: Excitation modes for Fourier transform ion cyclotron resonance mass spectrometry. J. Am. Soc. Mass Spectrom. 4, 433-452 (1993)

23. Heck, M., Blaum, K., Cakirli, R.B., Rodríguez, D., Schweikhard, L., Stahl, S., Ubieto-Díaz, M.: Dipolar and quadrupolar detection using an FT-ICR MS setup at the MPIK Heidelberg. Hyperfine Interact. 199, 347-355 (2011)

24. Tolmachev, A.V., Robinson, E.W., Wu, S., Kang, H., Lourette, N.M., Pasa-Tolic, L., Smith, R.D.: Trapped-ion cell with improved DC potential harmonicity for FT-ICR MS. J. Am. Soc. Mass Spectrom. 19, 586-597 (2008)

25. Tolmachev, A.V., Robinson, E.W., Wu, S., Smith, R.D., Futrell, J.H., PasaTolic, L.: Angular averaged profiling of the radial electric field in compensated FTICR Cells. J. Am. Soc. Mass Spectrom. 23, 1169-1172 (2012)

26. Brustkern, A.M., Rempel, D.L., Gross, M.L.: An electrically compensated trap designed to eighth order for FT-ICR mass spectrometry. J. Am. Soc. Mass Spectrom. 19, 1281-1285 (2008)

27. Bruce, J.E., Anderson, G.A., Lin, C.-Y., Gorshkov, M., Rockwood, A.L., Smith, R.D.: A novel high-performance Fourier transform ion cyclotron resonance cell for improved biopolymer characterization. J. Mass Spectrom. 35, 85-94 (2000)

28. Weisbrod, C.R., Kaiser, N.K., Skulason, G.E., Bruce, J.E.: Trapping ring electrode cell: a FTICR mass spectrometer cell for improved signal-to-noise and resolving power. Anal. Chem. 80, 6545-6553 (2008)

29. Boldin, I.A., Nikolaev, E.N.: Fourier transform ion cyclotron resonance cell with dynamic harmonization of the electric field in the whole volume by shaping of the excitation and detection electrode assembly. Rapid Commun. Mass Spectrom. 25, 122-126 (2011)

30. Popov, I.A., Nagornov, K., Vladimirov, G., Kostyukevich, Y.I., Nikolaev, E.N.: Twelve million resolving power on $4.7 \mathrm{~T}$ Fourier transform ion cyclotron resonance instrument with dynamically harmonized cell-observation of fine structure in peptide mass spectra. J. Am. Soc. Mass Spectrom. 25,1-10 (2014)

31. Nikolaev, E.N., Jertz, R., Grigoryev, A., Baykut, G.: Fine structure in isotopic peak distributions measured using a dynamically harmonized fourier transform ion cyclotron resonance cell at $7 \mathrm{~T}$. Anal. Chem. 84, $2275-2283$ (2012)

32. Nikolaev, E.N., Gorshkov, M.V., Mordehai, A.V., Talrose, V.L.: Ion cyclotron resonance signal-detection at multiples of the cyclotron frequency. Rapid Commun. Mass Spectrom. 4, 144-146 (1990)

33. Grosshans, P.B., Marshall, A.G.: General theory of excitation in ion cyclotron resonance mass spectrometry. Anal. Chem. 63, 2057-2061 (1991)

34. Knobler, M., Wanczek, K.P.: Detection of harmonics and multiples of the fundamental frequency in Fourier transform ion cyclotron resonance mass spectrometry. Proceedings of the 45th ASMS Conference on Mass Spectrometry and Allied Topics, Palm Springs, CA, June 1-5 (1997)

35. Nikolaev, E.N., Rakov, V.S., Futrell, J.H.: Analysis of harmonics for an elongated FTMS cell with multiple electrode detection. Int. J. Mass Spectrom. Ion Processes 157, 215-232 (1996)

36. Nagornov, K.O., Gorshkov, M.V., Kozhinov, A.N., Tsybin, Y.O.: Highresolution Fourier transform ion cyclotron resonance mass spectrometry with increased throughput for biomolecular analysis. Anal. Chem. 86, 9020-9028 (2014)

37. Gorshkov, M.V., Nikolaev, E.N.: Optimal cyclotron radius for high resolution FT-ICR spectrometry. Int. J. Mass Spectrom. Ion Processes 125, 1-8 (1993)

38. Miladinovic, S.M., Kozhinov, A.N., Tsybin, O.Y., Tsybin, Y.O.: Sidebands in Fourier transform ion cyclotron resonance mass spectra. Intl. J. Mass Spectrom 325, 10-18 (2012)

39. Kaiser, N., Quinn, J., Blakney, G., Hendrickson, C., Marshall, A.: A novel 9.4 Tesla FTICR mass spectrometer with improved sensitivity, mass resolution, and mass range. J. Am. Soc. Mass Spectrom. 22, 1343-1351 (2011)
40. Zhurov, K.O., Fornelli, L., Wodrich, M.D., Laskay, U.A., Tsybin, Y.O.: Principles of electron capture and transfer dissociation mass spectrometry applied to peptide and protein structure analysis. Chem. Soc. Rev. 42, 5014-5030 (2013)

41. Polfer, N.C.: Infrared multiple photon dissociation spectroscopy of trapped ions. Chem. Soc. Rev. 40, 2211-2221 (2011)

42. Fung, Y.M.E., Adams, C.M., Zubarev, R.A.: Electron ionization dissociation of singly and multiply charged peptides. J. Am. Chem. Soc. 131, 99779985 (2009)

43. Zubarev, R.A., Yang, H.: Multiple soft ionization of gas-phase proteins and swift backbone dissociation in collisions with $\leq 99 \mathrm{eV}$ electrons. Angew. Chem. Int. Ed. 49, 1439-1441 (2010)

44. Kaiser, N.K., McKenna, A.M., Savory, J.J., Hendrickson, C.L., Marshall, A.G.: Tailored ion radius distribution for increased dynamic range in FTICR mass analysis of complex mixtures. Anal. Chem. 85, 265-272 (2012)

45. Easterling, M.L., Mize, T.H., Amster, I.J.: Routine part-per-million mass accuracy for high-mass ions: space-charge effects in MALDI FT-ICR. Anal. Chem. 71, 624-632 (1999)

46. Schaub, T.M., Hendrickson, C.L., Horning, S., Quinn, J.P., Senko, M.W., Marshall, A.G.: High-Performance mass spectrometry: Fourier transform ion cyclotron resonance at 14.5 Tesla. Anal. Chem. 80, 3985-3990 (2008)

47. Barry, J.A., Robichaud, G., Muddiman, D.C.: Mass Recalibration of FTICR mass spectrometry imaging data using the average frequency shift of ambient ions. J. Am. Soc. Mass Spectrom. 24, 1137-1145 (2013)

48. Williams, D.K., Muddiman, D.C.: Parts-per-billion mass measurement accuracy achieved through the combination of multiple linear regression and automatic gain control in a Fourier transform ion cyclotron resonance mass spectrometer. Anal. Chem. 79, 5058-5063 (2007)

49. Masselon, C., Tolmachev, A.V., Anderson, G.A., Harkewicz, R., Smith, R.D.: Mass measurement errors caused by "local" frequency perturbations in FTICR mass spectrometry. J. Am. Soc. Mass Spectrom. 13, 99-106 (2002)

50. Kilgour, D.P.A., Wills, R., Qi, Y.L., O'Connor, P.B.: Autophaser: an algorithm for automated generation of absorption mode spectra for FTICR MS. Anal. Chem. 85, 3903-3911 (2013)

51. Xian, F., Hendrickson, C.L., Blakney, G.T., Beu, S.C., Marshall, A.G.: Automated broadband phase correction of Fourier transform ion cyclotron resonance mass spectra. Anal. Chem. 82, 8807-8812 (2010)

52. Kilgour, D.P.A., Nagornov, K.O., Kozhinov, A.N., Zhurov, K.O., Tsybin, Y.O.: Producing absorption mode FT-ICR mass spectra with non-quadratic phase correction functions (manuscript submitted)

53. He, F., Hendrickson, C.L., Marshall, A.G.: Baseline mass resolution of peptide isobars: a record for molecular mass resolution. Anal. Chem. 73, $647-650$ (2000)

54. Schouwey, C., Holstein, J.J., Scopelliti, R., Zhurov, K.O., Nagornov, K.O., Tsybin, Y.O., Smart, O.S., Bricogne, G., Severin, K.: Self-assembly of a giant molecular Solomon link from 30 subcomponents. Angew. Chem. Int. Ed. 53, 11261-11265 (2014)

55. Schweikhard, L., Lindinger, M., Kluge, H.J.: Quadrupole-detection FTICR mass spectrometry. Int. J. Mass Spectrom. Ion Processes 98, 25-33 (1990)

56. Nagornov, K.O., Kozhinov, A.N., Zhurov, K.O., Tsybin, Y.O.: Mapping ion cloud dynamics reveals optimal regimes in FT-ICR MS. Proceedings of the 62nd American Society for Mass Spectrometry Conference on Mass Spectrometry and Allied Topics, Baltimore, MD, June 15-19 (2014)

57. Chingin, K., Chen, H., Gamez, G., Zenobi, R.: Exploring fluorescence and fragmentation of ions produced by electrospray ionization in ultrahigh vacuum. J. Am. Soc. Mass Spectrom. 20, 1731-1738 (2009)

58. Frankevich, V., Chagovets, V., Widjaja, F., Barylyuk, K., Yang, Z., Zenobi, R.: Fluorescence resonance energy transfer of gas-phase ions under ultra high vacuum and ambient conditions. Phys. Chem. Chem. Phys 16, 8911-8920 (2014)

59. von Lindenfels, D., Vogel, M., Quint, W., Birkl, G., Wiesel, M.: Half-open Penning trap with efficient light collection for precision laser spectroscopy of highly charged ions. Hyperfine Interact. 227, 197-207 (2014)

60. Kozhinov, A.N., Nagornov, K.O., Ayoub, D., Tsybin, Y.O.: Unexplored reserves of resolution in Fourier transform mass spectrometry. Proceedings of the 62nd American Society for Mass Spectrometry Conference on Mass Spectrometry and Allied Topics, Baltimor, MD, June 15-19 (2014) 